\title{
FINITELY GENERATED COTORSION MODULES
}

\author{
EDGAR E. ENOCHS ${ }^{1}$, J. R. GARCIA ROZAS ${ }^{2}$ AND LUIS OYONARTE ${ }^{2}$ \\ ${ }^{1}$ Department of Mathematics, University of Kentucky, Lexington, \\ KY 40506-0027, USA (enochs@ms.uky.edu) \\ ${ }^{2}$ Departamento de Álgebra y Análisis Matemático, Universidad de Almería, \\ 04120 Almería, Spain (jrgrozas@ualm.es; oyonarte@ualm.es)
}

(Received 15 June 1999)

\begin{abstract}
We describe the structure of finitely generated cotorsion modules over commutative noetherian rings. Also we characterize the so-called covering morphisms between finitely generated modules over these rings.
\end{abstract}

Keywords: noetherian; module; flat; cotorsion; cover; complete

AMS 2000 Mathematics subject classification: Primary 16D10

Secondary $13 \mathrm{C} 11$

\section{Introduction}

The study of torsion-free covers began in [3], where it was proved that any module over an integral domain has a torsion-free cover with respect to the usual torsion theory. Later, other authors studied this type of problem in more general situations. For example, Teply extended this result to the case of torsion-free covers relative to arbitrary hereditary torsion theories (see $[\mathbf{1 1}, \mathbf{1 4}-\mathbf{1 6}]$ ).

Covers have been studied for arbitrary classes of modules (see [5]) with the class of flat modules and the class of submodules of flat modules being two of the most important such classes. Gómez and Torrecillas [12] study the existence of covers by submodules of flat modules. In [17] Xu proved that any module over a commutative noetherian ring of finite Krull dimension has a flat cover (see also [18, Theorem 4.3.5]). We note that the existence of flat covers over any ring was conjectured by Enochs [5, p. 196], and this conjecture has recently been positively settled by Bican et al. [2] .

Wakamatsu's Lemma [18, Lemma 2.1.1] implies that kernels of flat covers are cotorsion. So cotorsion modules are important in the study of flat covers. The structure of flat cotorsion modules over commutative noetherian rings is known [18, Theorem 4.1.15]. In this paper we give a complete structure theorem for finitely generated cotorsion modules (Corollary 2.5). Section 3 is devoted to the study of covering morphisms.

The notion of a covering morphism was introduced in [9] and those morphisms were studied in $[\mathbf{1 0}]$ and $[\mathbf{8}]$. They are useful in developing a categorical theory analogous to 
the Galois theory of fields. In this paper we use our structure results to characterize covering morphisms between finitely generated modules over commutative noetherian rings.

Most of the results we give in the paper will make use of the techniques exposed by $\mathrm{Xu}$ in $[\mathbf{1 8}, \S 4.1]$. These techniques are valid when the base ring is commutative noetherian, therefore, and for convenience, throughout this paper $R$ will be a non-zero commutative noetherian ring and all modules will be unital $R$-modules (unless otherwise stated).

We recall that a module $C$ is said to be cotorsion if $\operatorname{Ext}^{1}(F, C)=0$ for all flat modules $F$. For a module $M, \operatorname{PE}(M)$ will denote its pure injective envelope. The symbol $\Omega$ will mean the set of all maximal ideals of $R$, while $\operatorname{Spec}(R)$ will be the set of all prime ideals of $R$. If $M$ is any module, we let $\operatorname{rad}(M)$ denote the Jacobson radical of $M$.

Given $R$, we will consider the ring $\hat{R}=\prod_{\eta \in \Omega} \hat{R}_{\eta}$. This is the completion of $R$ with respect to the topology having all finite products $\eta_{1} \eta_{2} \cdots \eta_{s}\left(\eta_{1}, \ldots, \eta_{s} \in \Omega, s \geqslant 1\right)$ as a fundamental system of neighbourhoods of zero. $\hat{R}$ will be noetherian if and only if $\Omega$ is finite. We recall that $\operatorname{PE}(R)=\hat{R}$ [18, Corollary 4.2.4]. If $\Omega^{\prime} \subseteq \Omega$, we will identify $\prod_{\eta \in \Omega^{\prime}} \hat{R}_{\eta}$ with a subset of $\hat{R}=\prod_{\eta \in \Omega} \hat{R}_{\eta}$ in the obvious manner.

Given a class of modules $\mathcal{F}$, and a module $M$, an $\mathcal{F}$-cover is a module $F \in \mathcal{F}$ together with a morphism $\varphi: F \rightarrow M$ such that

(i) for any $F^{\prime} \in \mathcal{F}$ and any $f: F^{\prime} \rightarrow M$, there exists $g: F^{\prime} \rightarrow F$ with $\varphi \circ g=f$; and

(ii) for any $g: F \rightarrow F$ with $\varphi \circ g=\varphi, g$ is an automorphism.

When $F$ and $\varphi$ are such that (i) holds and perhaps (ii) does not, then $\varphi: F \rightarrow M$ is an $\mathcal{F}$-precover.

We note that $\mathcal{F}$-covers are unique up to isomorphism. Also, if $\psi: G \rightarrow M$ is an $\mathcal{F}$ precover and $\varphi: F \rightarrow M$ an $\mathcal{F}$-cover, then any $f: F \rightarrow G$ such that $\psi f=\varphi$ maps $F$ isomorphically onto a direct summand of $G$. And any $g: G \rightarrow F$ with $\varphi g=\psi$ is surjective and ker $g$ is a direct summand of $G$.

A flat cover is an $\mathcal{F}$-cover, where $\mathcal{F}$ is the class of flat modules. We will follow this convention in terminology for other such classes $\mathcal{F}$. For example, if $\mathcal{F}$ is the class of projective modules, an $\mathcal{F}$-cover is a projective cover.

\section{The main theorem}

This section is devoted to the study of the structure of finitely generated cotorsion modules.

Pure injective modules are cotorsion and so give a supply of cotorsion modules. If $E$ is any injective $R$-module, $\operatorname{Hom}_{R}(M, E)$ is pure injective for any module $M$. If $S \leqslant N$ is pure, $M \otimes S \rightarrow M \otimes N$ is an injection. Hence $\operatorname{Hom}(M \otimes N, E) \rightarrow \operatorname{Hom}(M \otimes S, E)$ and so $\operatorname{Hom}(N, \operatorname{Hom}(M, E)) \rightarrow \operatorname{Hom}(S, \operatorname{Hom}(M, E))$ are surjective. Also if $R \rightarrow R^{\prime}$ is any ring homomorphism, then any pure injective $R^{\prime}$-module is pure injective as an $R$-module. If $p \in \operatorname{Spec}(R)$, then $\hat{R}_{p}$ is a flat $R$-module, hence any pure injective $\hat{R}_{p}$-module is pure injective as an $R$-module. In particular, if $M$ is a finitely generated $\hat{R}_{p}$-module, then $M$ is Matlis reflexive as an $\hat{R}_{p}$-module and so has the form $\operatorname{Hom}_{\hat{R}_{p}}\left(N, E_{\hat{R}_{p}}(k(p))\right)$, where 
$k(p)$ is the residue field of $\hat{R}_{p}$ and $N$ is some $\hat{R}_{p}$-module. Hence $M$ is pure injective as an $\hat{R}_{p}$-module and so as an $R$-module. More generally, any finitely generated module over $\hat{R}_{p_{1}} \times \cdots \times \hat{R}_{p_{s}}$ for $p_{1}, \ldots, p_{s} \in \operatorname{Spec}(R)$ is pure injective as an $R$-module, and so cotorsion as an $R$-module.

We note that if $F$ is flat then $F$ is pure injective if and only if $F$ is cotorsion. For pure injective implies cotorsion for any module. Now suppose $F$ is flat and cotorsion. Since $F \leqslant \mathrm{PE}(F)$ is pure and $\mathrm{PE}(F)$ is flat by $[\mathbf{1 8}$, Lemma 3.1.6], we have that $\mathrm{PE}(F) / F$ is flat (also by $[\mathbf{1 8}$, Lemma 3.1.6]). But $F$ is cotorsion, so $0 \rightarrow F \rightarrow \mathrm{PE}(F) \rightarrow \mathrm{PE}(F) / F \rightarrow 0$ splits and then $F$, as a direct summand of $\mathrm{PE}(F)$, is pure injective (so in fact $F=\mathrm{PE}(F)$ ).

Proposition 2.1. Let $M$ be an $R$-module. If $M$ is finitely generated, then a linear $R \rightarrow M$ has at most one extension $\hat{R} \rightarrow M$. If $M$ (whether finitely generated or not) is such that every linear $R \rightarrow M$ has a unique extension $\hat{R} \rightarrow M$, then $M$ can be made into an $\hat{R}$-module with the scalar multiplication extending the original scalar multiplication in a unique fashion.

Proof. If $M$ is finitely generated and some $R \rightarrow M$ has two distinct extensions $\hat{R} \rightarrow M$, then there would exist a non-zero map $\hat{R} / R \rightarrow M$ and so there is an exact $\hat{R} / R \rightarrow N \rightarrow 0$ with $N \leqslant M, N \neq 0$. Note also that $\bar{\eta} N \neq N$ for some $\bar{\eta} \in \Omega$. Now

$$
\bar{\eta} \hat{R}=\bar{\eta} \prod \hat{R}_{\eta}=\prod \bar{\eta} \hat{R}_{\eta}=\bar{\eta} \hat{R}_{\bar{\eta}} \times \prod_{\eta \neq \bar{\eta}} \hat{R}_{\eta}
$$

Hence $\bar{\eta} \hat{R}+R=\hat{R}$ (recall that $R / \eta \rightarrow \hat{R}_{\eta} / \eta \hat{R}_{\eta}$ is an isomorphism) and so $\bar{\eta}(\hat{R} / R)=\hat{R} / R$. But this is not compatible with the above.

Now assume $M$ is arbitrary and that every $R \rightarrow M$ has a unique extension $\hat{R} \rightarrow M$.

Given any $r \in \hat{R}$ and any $x \in M$, we define $r x$ as follows: we consider the $R$-homomorphism $R \stackrel{\cdot x}{\longrightarrow} M$, and we extend it to $\hat{R} \rightarrow M$. So $r x$ is the image of $r$ by the last map. Then if $r, s \in \hat{R}$ and $x \in M$ we consider the situation

$$
\hat{R} \stackrel{\cdot s}{\rightarrow} \hat{R} \stackrel{\cdot r}{\rightarrow} \hat{R} \stackrel{\cdot x}{\rightarrow} M .
$$

If we compose the last three homomorphisms in the two possible ways, and we apply both compositions to $1 \in \hat{R}$, we get the equality $(r s) x=r(s x)$. The rest of the module axioms are trivial.

Proposition 2.2. If $M$ is an $\hat{R}$-module that is finitely generated as an $R$-module, then $\left(\prod_{\eta \in \Omega^{\prime}} \hat{R}_{\eta}\right) M=0$ for some $\Omega^{\prime} \subseteq \Omega$ with $\Omega \backslash \Omega^{\prime}$ finite, and $M$ is a cotorsion $R$-module.

Proof. We first argue that if $M$ is an $\hat{R}$-module that is finitely generated as an $R$ module and if $\left(\oplus_{\eta \in \Omega} \hat{R}_{\eta}\right) M=0$, then $M=0$.

For if $M \neq 0$ there is an $\bar{\eta} \in \Omega$ such that $\bar{\eta} M \neq M$. Since

$$
\bar{\eta} \hat{R}=\bar{\eta} \prod_{\eta \in \Omega} \hat{R}_{\eta}=\prod_{\eta \in \Omega} \bar{\eta} \hat{R}_{\eta}=\bar{\eta} \hat{R}_{\bar{\eta}} \times \prod_{\eta \neq \bar{\eta}} \hat{R}_{\eta}
$$


we see that $\bar{\eta}\left(\hat{R} / \oplus_{\eta \in \Omega} \hat{R}_{\eta}\right)=\hat{R} / \oplus_{\eta \in \Omega} \hat{R}_{\eta}$. But if $\left(\oplus_{\eta \in \Omega} \hat{R}_{\eta}\right) M=0$, then $M$ is an $\left(\hat{R} / \oplus_{\eta \in \Omega} \hat{R}_{\eta}\right)$-module, which is impossible.

Therefore, if $M$ is an $\hat{R}$-module which is finitely generated as an $R$-module, then $\left(\oplus_{\eta \in \Omega} \hat{R}_{\eta}\right) M=M$ by applying the above to $N=M /\left(\oplus_{\eta \in \Omega} \hat{R}_{\eta} M\right)$.

Now we see that since $M$ is finitely generated, $M=\left(\oplus_{i=1}^{s} \hat{R}_{\eta_{i}}\right) M$ for some finite number of distinct $\eta_{1}, \eta_{2}, \ldots, \eta_{s} \in \Omega$. Let $e \in \oplus_{i=1}^{s} \hat{R}_{\eta_{i}}$ be $(1,1, \ldots, 1)$. Then $e x=x$ for all $x \in M$ and then $(1-e) x=0 \forall x \in M$. Thus, $\left(\prod_{\eta \in \Omega^{\prime}} \hat{R}_{\eta}\right) M=0$, where $\Omega \backslash \Omega^{\prime}=\left\{\eta_{1}, \eta_{2}, \ldots, \eta_{s}\right\}$.

It is easy to observe that $M$ is a finitely generated

$$
\hat{R} / \prod_{\eta \in \Omega^{\prime}} \hat{R}_{\eta} \cong \hat{R}_{\eta_{1}} \times \cdots \times \hat{R}_{\eta_{s}} \text {-module; }
$$

so $M$ is a cotorsion $R$-module.

We now collect our results in the next theorem.

Theorem 2.3. Let $M$ be a finitely generated $R$-module. Then $M$ is cotorsion if and only if every linear $R \rightarrow M$ has an $R$-linear extension $\hat{R} \rightarrow M$. Also $M$ is cotorsion if and only if $M$ is an $\hat{R}$-module with the scalar multiplication extending the original scalar multiplication by $R$. When this is the case, the $\hat{R}$-module structure is unique and for some $\Omega^{\prime} \subseteq \Omega$ with $\Omega \backslash \Omega^{\prime}$ finite, $\left(\prod_{\eta \in \Omega^{\prime}} \hat{R}_{\eta}\right) M=0$ and so $M$ is an $\hat{R}_{\eta_{1}} \times \cdots \times \hat{R}_{\eta_{s}}$-module with $\Omega \backslash \Omega^{\prime}=\left\{\eta_{1}, \ldots, \eta_{s}\right\}$ and where the $\eta_{i}$ are distinct.

Proof. If $M$ is cotorsion, any $R \rightarrow M$ has an $R$-linear extension $\hat{R} \rightarrow M$ (since $\hat{R} / R$ is flat), which is unique by Proposition 2.1. Then, also by Proposition 2.1, $M$ is uniquely an $\hat{R}$-module with scalar multiplication extending the original scalar multiplication. By Proposition 2.2 we get the $\Omega^{\prime} \subseteq \Omega$ with $\Omega \backslash \Omega^{\prime}$ finite and such that $\left(\prod_{\eta \in \Omega^{\prime}} \hat{R}_{\eta}\right) M=0$.

Conversely, if every $R \rightarrow M$ has an extension $\hat{R} \rightarrow M$, this extension is unique (Proposition 2.1), and so $M$ is an $\hat{R}$-module, also by Proposition 2.1. Then Proposition 2.2 shows that $M$ is cotorsion. If we assume already that $M$ is an $\hat{R}$-module (again with scalar multiplication extending that by $R$ ), Proposition 2.2 gives that $M$ is cotorsion.

Corollary 2.4. Let $C \rightarrow M \rightarrow 0$ be exact, where $C$ is cotorsion and $M$ is finitely generated. Then $M$ is cotorsion.

Proof. Any linear $R \rightarrow M$ lifts to $R \rightarrow C$. Such an $R \rightarrow C$ has an extension $\hat{R} \rightarrow C$ which gives an extension $\hat{R} \rightarrow C \rightarrow M$ of $R \rightarrow M$.

Corollary 2.5. Let $M$ be any finitely generated cotorsion $R$-module. Then $M=M_{1} \times$ $\cdots \times M_{s}$ uniquely, where $M_{i}$ is a finitely generated $\hat{R}_{\eta_{i}}$-module for $\eta_{i} \in \Omega, i=1, \ldots, s$, and $\eta_{i} \neq \eta_{j}$ whenever $i \neq j$.

Proof. The proof follows immediately from Theorem 2.3.

Lemma 2.6. Let $M$ be an $R$-module such that every $R \rightarrow M$ has a unique extension $\hat{R} \rightarrow M$ (so $M$ is an $\hat{R}$-module by Proposition 2.1). Then if $N$ is an $\hat{R}$-module and if $f: N \rightarrow M$ is $R$-linear, then $f$ is $\hat{R}$-linear. 
Proof. Let $y \in N$. Consider the map $\hat{R} \rightarrow M$, namely $r \mapsto f(r y)-r f(y)$. This map is zero on $R$, so by hypothesis is zero on $\hat{R}$. Hence $f$ is $\hat{R}$-linear.

We now consider closure operations for the class of finitely generated cotorsion modules.

Proposition 2.7. Let $M$ be a finitely generated $R$-module. If $S \leqslant M$ is a submodule, then $M$ is cotorsion if and only if $S$ and $M / S$ are cotorsion.

Proof. If $S$ and $M / S$ are cotorsion, we have the exact

$$
0=\operatorname{Ext}^{1}(F, S) \rightarrow \operatorname{Ext}^{1}(F, M) \rightarrow \operatorname{Ext}^{1}(F, M / S)=0
$$

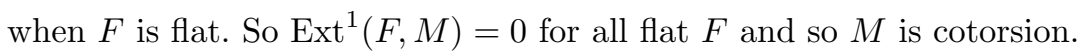

Conversely, suppose $M$ is cotorsion. By Corollary 2.4, $M / S$ is cotorsion, and then by Theorem 2.3 and Lemma $2.6 M$ and $M / S$ are $\hat{R}$-modules and $M \rightarrow M / S$ is $\hat{R}$-linear. Hence $S$ is an $\hat{R}$-submodule of $M$ and so, again by Theorem 2.3 , is cotorsion.

If we let $t(M)=\{x \in M ; R x$ is cotorsion $\}$, we immediately see that $t(M)$ is a submodule of $M$, and so it is a finitely generated submodule. Therefore, $t(M)=R x_{1}+\cdots+R x_{n}$ for some $x_{1}, \ldots, x_{n} \in M$, and $R x_{i}$ are all cotorsion. Then, using the fact that quotients and finite direct sums of cotorsion modules are cotorsion, we get that $t(M)$ is a cotorsion submodule of $M$. In fact, it is clear that it is the largest cotorsion submodule of $M$. If we now observe that $t(M / t(M))=0$, we see that $t$ defines a left exact radial and hence we have an associated torsion theory [13]. With $r x \cong R / I$ and $I=\operatorname{Ann}(x)$, we ask: for which ideals $I \leqslant R$ is $R / I$ cotorsion?

Proposition 2.8. If $R$ is of finite Krull dimension and $I \leqslant R$ is an ideal, then $R / I$ is a cotorsion $R$-module if and only if $R / I$ is a complete semilocal ring.

Proof. Let $R / I$ be a cotorsion $R$-module. Then $R / I$ is an $\hat{R}$-module and so an $(\hat{R} / I \hat{R})$-module. But clearly $\hat{R} / I \hat{R}=\widehat{R / I}$; so, by Theorem $2.3, R / I$ is a cotorsion $R / I$ module. Since $R / I$ is a flat $R / I$-module, $R / I$ is also a pure injective $R / I$-module, so $R / I=P E_{R / I}(R / I)$. But by $\left[\mathbf{1 8}\right.$, Corollary 4.2.4], $P E_{R / I}(R / I)=\prod(\widehat{R / I})_{\eta / I}$ with the product over the maximal ideals $\eta / I$ of $R / I$. Since $R / I$ is noetherian, we see that there are only a finite number of such maximal ideals $\eta / I$ (because otherwise $\oplus(\widehat{R / I})_{\eta / I}$ is not finitely generated). Hence $R / I$ is a complete semilocal ring.

Conversely, if $R / I$ is a complete semilocal ring, then $R / I=P E_{R / I}(R / I)$ and so $R / I$ is a pure injective, hence cotorsion, $R / I$-module. But then by [18, Proposition 3.3.3], $R / I$ is a cotorsion $R$-module.

\section{Covers and coverings by flat modules}

Let $\mathcal{F}$ be a class of modules. A homomorphism of $R$-modules $f: M_{1} \rightarrow M_{2}$ is said to be an $\mathcal{F}$-covering morphism if, whenever we take arbitrary $\mathcal{F}$-covers $\varphi_{1}: F_{1} \rightarrow M_{1}$ and $\varphi_{2}: F_{2} \rightarrow M_{2}$ of $M_{1}$ and $M_{2}$, respectively, every induced morphism $g: F_{1} \rightarrow F_{2}$ with $\varphi_{2} \circ g=f \circ \varphi_{1}$ is an isomorphism. We note that every such $g$ is an isomorphism if and only if one of them is (see [9] for details about covering morphisms). In this section we 
will give several characterizations of $\mathcal{F}$-covering morphisms between finitely generated modules by restricting ourselves to the case in which $\mathcal{F}$ is the class of all flat modules.

Covering morphisms (with respect to the class of flat modules) of finitely generated modules over a principal ideal domain have been characterized in [9] as those epimorphism whose kernel is superfluous.

Proposition 3.1. Let $M$ be a finitely generated cotorsion module. Let $\eta_{1}, \ldots, \eta_{s}$ be distinct maximal ideals of $R$ such that $\left(\prod_{\eta \in \Omega^{\prime}} \hat{R}_{\eta}\right) M=0$, where $\Omega^{\prime}=\Omega \backslash\left\{\eta_{1}, \ldots, \eta_{s}\right\}$; so $M$ is an $\hat{R}_{\eta_{1}} \times \cdots \times \hat{R}_{\eta_{s}}$-module. Let $M=M_{1} \times \cdots \times M_{s}$ be the corresponding decomposition of $M$ (so $M_{i}$ is a finitely generated $\hat{R}_{\eta_{i}}$-module). If $\hat{R}_{\eta_{i}}^{n_{i}} \rightarrow M_{i}$ is a projective cover of $M_{i}$ as an $\hat{R}_{\eta_{i}}$-module, then $\varphi: F=\hat{R}_{\eta_{1}}^{n_{1}} \times \cdots \times \hat{R}_{\eta_{s}}^{n_{s}} \rightarrow M_{1} \times \cdots \times M_{s}=M$ is a flat cover of $M$ as an $R$-module.

Proof. $F$ is a flat $R$-module and $\varphi$ is surjective. If $K=\operatorname{ker} \varphi=K_{1} \times \cdots \times K_{s}$, then $K_{i}$ is a finitely generated $\hat{R}_{\eta_{i}}$-module. Then $K_{i}$ is a cotorsion $R$-module and so also is $K=K_{1} \times \cdots \times K_{s}$. Therefore, if $G$ is flat we have that $\operatorname{Hom}(G, F) \rightarrow \operatorname{Hom}(G, M) \rightarrow$ $\operatorname{Ext}^{1}(G, K)=0$ is exact and then $\varphi: F \rightarrow M$ is a flat precover.

The fact that it is a flat cover results from the fact that any $R$-linear $\hat{R}_{\eta_{i}}^{n_{i}} \rightarrow \hat{R}_{\eta_{i}}^{n_{i}}$ is also $\hat{R}_{\eta_{i}}$-linear and that $\hat{R}_{\eta_{i}}^{n_{i}} \rightarrow M_{i}$ is a projective cover as $\hat{R}_{\eta_{i}}$-modules.

Theorem 3.2. Any finitely generated $R$-module has a flat and cotorsion cover.

Proof. Let $M$ be a finitely generated $R$-module. We consider $t(M) \subseteq M$ the sum of all cotorsion submodules of $M$ and $F \rightarrow t(M)$ a flat cover. Then $F$ is flat and cotorsion [6, p. 182, Corollary]. We prove that $F \rightarrow t(M) \hookrightarrow M$ is the flat and cotorsion cover of $M$.

Given $h: G \rightarrow M$ with $G$ flat cotorsion, then $h(G)$ is cotorsion so $h(G) \subseteq t(M)$. Hence, there exists $g: G \rightarrow F$ such that

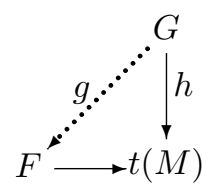

is commutative. So $F \rightarrow M$ is a precover.

Finally, let $d: F \rightarrow F$ be a map such that the triangle

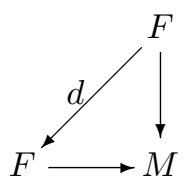

is commutative. Then it is easy to see that the triangle 


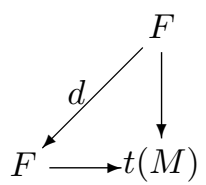

is commutative too and so $d$ is an automorphism.

Corollary 3.3. The flat and cotorsion cover of a finitely generated module $M$ is a map of the form $\hat{R}_{\eta_{1}}^{n_{1}} \times \cdots \times \hat{R}_{\eta_{s}}^{n_{s}} \rightarrow M$ for some natural numbers $n_{1}, \ldots, n_{s}$ and maximal ideals $\eta_{1}, \ldots, \eta_{s}$.

Proof. Since $M$ is finitely generated, $t(M)$ is finitely generated too, so it has a decomposition $t(M)=A_{1} \times \cdots \times A_{s}$, where $A_{i}$ is a finitely generated $\hat{R}_{\eta_{i}}$-module and $\eta_{i}$ is a maximal ideal of $R$ for each $i$ (Corollary 2.5). Then, by Proposition 3.1 we have that $\hat{R}_{\eta_{i}}^{n_{i}} \rightarrow A_{i}$ is a flat cover of $A_{i}$ as an $R$-module (recall that every finitely generated $\hat{R}_{\eta_{i}}$-module has a projective cover which is free, so it is of the form $\left.\hat{R}_{\eta_{i}}^{n_{i}} \rightarrow A_{i}\right)$. Hence, $\hat{R}_{\eta_{i}}^{n_{i}} \times \cdots \times \hat{R}_{\eta_{s}}^{n_{s}} \rightarrow t(M)$ is a flat cover (as $R$-modules) and, by the proof of Theorem 3.2, $\hat{R}_{\eta_{i}}^{n_{i}} \times \cdots \times \hat{R}_{\eta_{s}}^{n_{s}} \rightarrow t(M) \hookrightarrow M$ is a flat and cotorsion cover (as $R$-modules).

Remark 3.4. Since the class of flat and cotorsion modules is not closed under arbitrary direct sums it is clear that there are modules without flat and cotorsion covers. For example, take $(R, \eta)$ a non-complete local ring and consider $G=\oplus_{\mathbb{N}} \widehat{R}$ a countable direct sum of copies of the completion of $R$ with respect to the $\eta$-adic topology. Then $G$ is flat but it is not cotorsion (it is not pure-injective). If $G$ had a flat and cotorsion cover $F \rightarrow G$ then the canonical injections $\hat{R} \rightarrow G$ would factorize through $F$. So by the universal property of the direct sum we would find a map $G \rightarrow F$ such that the composition $G \rightarrow F \rightarrow G$ is the identity. So $F \rightarrow G$ would split and so $G$ would be cotorsion, a contradiction.

We will now study covering morphisms $M_{1} \rightarrow M_{2}$ between finitely generated $R$ modules.

Proposition 3.5. If $f: M_{1} \rightarrow M_{2}$ is a covering homomorphism, where $M_{1}$ is finitely generated, then $f$ is surjective and ker $f$ is cotorsion.

Proof. If $\varphi_{i}: F_{i} \rightarrow M_{i}$ is a flat cover for $i=1,2$, then each $\varphi_{i}$ is surjective (any $R \rightarrow M_{i}$ can be factored $R \rightarrow F_{i} \rightarrow M_{i}$ ). By hypothesis there is a commutative diagram

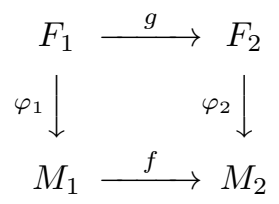

with $F_{1} \stackrel{g}{\rightarrow} F_{2}$ an isomorphism. Hence $f$ must be a surjection. But we also have a surjection $g^{-1}\left(\operatorname{ker} \varphi_{2}\right) \rightarrow \operatorname{ker} f$ that agrees with $\varphi_{1}$, and $\operatorname{ker} \varphi_{2}$ is cotorsion by Wakamatsu's Lemma. Therefore, since $g^{-1}\left(\operatorname{ker} \varphi_{2}\right) \cong \operatorname{ker} \varphi_{2}$, we get, by Corollary 2.4 , that $\operatorname{ker} f$ is cotorsion. 
The fact that ker $f$ is cotorsion also follows from a more general fact proved in $[\mathbf{9}$, Theorem 2.7].

Proposition 3.6. Let $M$ be a finitely generated $R$-module. If $N$ is a submodule of $M$ and the projection $M \rightarrow M / N$ is a covering morphism, then for any submodule $K \leqslant N$ the projections $M \rightarrow M / K$ and $M / K \rightarrow M / N$ are both covering morphisms.

Proof. If $M \rightarrow M / N$ is covering, we know that $N$ is cotorsion, and since it is finitely generated, any $K \leqslant N$ is also cotorsion by Proposition 2.7. Suppose now that $G \rightarrow M / K$, $F \rightarrow M / N$ and $F \rightarrow M$ are flat covers and consider the induced commutative diagram

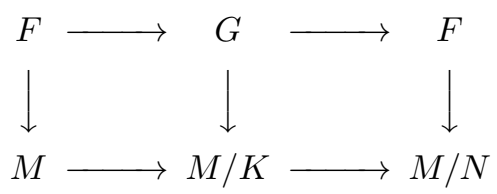

Since $M \rightarrow M / N$ is covering, $F \rightarrow G \rightarrow F$ is an isomorphism and then $F \rightarrow G$ is a monomorphism, so we can suppose $F \leqslant G$. Now, from the properties of a cover, it is easy to see that if the restriction of a flat cover $G \rightarrow M / K$ to a flat $F \leqslant G$ gives a flat precover $F \rightarrow M / K$, then $F=G$. And it is not hard to prove that $F \rightarrow M \rightarrow M / K$ is a flat precover since $K$ is cotorsion, so we have that the original $F \rightarrow G$ is an isomorphism. Thus, $M \rightarrow M / K$ is covering.

Of course if $F \rightarrow G \rightarrow F$ and $F \rightarrow G$ are both isomorphisms, then $G \rightarrow F$ is also an isomorphism and so $M / K \rightarrow M / N$ is covering.

Theorem 3.7. Let $M_{1}, M_{2}$ be any two finitely generated modules and $f: M_{1} \rightarrow M_{2}$ any homomorphism of $R$-modules. The following statements are equivalent.

(1) $f$ is covering.

(2) $f$ is surjective, $C=\operatorname{ker} f$ is cotorsion and for any submodule $D \leqslant C$, the module $C / D$ does not contain non-zero direct summands of $M_{1} / D$.

(3) $f$ is surjective, $C$ is cotorsion and for any maximal $D \leqslant C$, the module $C / D$ is not a direct summand of $M_{1} / D$.

Proof. (1) $\Rightarrow(2)$ We know that $f$ is surjective and that $C$ is cotorsion.

If $D \leqslant C$ is any submodule, the induced morphism $M_{1} / D \rightarrow M_{2}$ is covering by Proposition 3.6, and $\left[\mathbf{9}\right.$, Proposition 2.8] says then that $\operatorname{ker}\left(M_{1} / D \rightarrow M_{2}\right)=C / D$ contains no non-zero direct summands of $M_{1} / D$ (we note that this is easy to argue directly).

$(2) \Rightarrow(3)$ Clear.

(3) $\Rightarrow(1)$ Let $\phi: F \rightarrow M_{2}$ be a flat cover. Since $f$ is surjective and $C$ is cotorsion, we get a morphism $\varphi: F \rightarrow M_{1}$ such that $f \varphi=\phi$. Let us prove that $\varphi$ is surjective. This is clearly equivalent to the condition $C \subseteq \operatorname{Im} \varphi$. We consider $F^{\prime}=\varphi^{-1}(C)$, and we want to prove that $\varphi^{\prime}=\left.\varphi\right|_{F^{\prime}}: F^{\prime} \rightarrow C$ is surjective. But the latter is equivalent to proving that the map $F^{\prime} \stackrel{\varphi^{\prime}}{\longrightarrow} C \rightarrow C / D$ is surjective for every maximal $D \leqslant C$. Suppose that this is 
not the case. Then there exists a maximal $D$ such that the map $F^{\prime} \stackrel{\varphi^{\prime}}{\longrightarrow} C \rightarrow C / D$ is zero (because $C / D$ is simple), and then $\operatorname{Im} \varphi^{\prime} \subseteq D$. This implies that $\operatorname{Im}(\varphi) \cap C \subseteq D$, and it is clear that $\operatorname{Im}(\varphi)+C=M_{1}$ since $f \varphi=\phi$ is surjective. So we get that $M_{1} / D$ is the direct sum of $(\operatorname{Im}(\varphi)+D) / D$ and $C / D$, which contradicts the hypothesis.

Let us prove now that $\varphi: F \rightarrow M_{1}$ is a flat precover. Suppose then that $G$ is a flat module and that $h: G \rightarrow M_{1}$ is any homomorphism. Since $\phi$ is a flat cover of $M_{2}$, we see that there exists a morphism $g: G \rightarrow F$ such that $\phi g=f h$. Consider now the map $h-\varphi g: G \rightarrow C$. If we find a morphism $t: G \rightarrow F^{\prime}$ such that $\varphi^{\prime} t=h-\varphi g$, we will see that, if we let $k: F^{\prime} \rightarrow F$ be the inclusion, then $\varphi k t=h-\varphi g$, and so $\varphi(k t+g)=h$, where $k t+g: G \rightarrow F$. Therefore, we will have shown that $\varphi: F \rightarrow M_{1}$ is a flat precover.

Thus, we will prove that for any morphism $G \stackrel{\alpha}{\rightarrow} C$ there exists a map $G \stackrel{\beta}{\rightarrow} F^{\prime}$ such that $\alpha=\varphi^{\prime} \beta$. It is clear that it suffices to argue that this is the case when $\alpha$ is a flat cover. But $C$ is finitely generated and cotorsion, so the flat cover of $C$ is a finite direct sum of $\hat{R}_{\eta}$ for some maximal ideals $\eta$ of $R$ (Proposition 3.1). Hence we can suppose $\alpha: \hat{R}_{\eta} \rightarrow C$ for some $\eta \in \Omega$.

We can also extend $\alpha: \hat{R}_{\eta} \rightarrow C$ to an $R$-linear $\alpha: \hat{R} \rightarrow C$ (letting the map be zero on $\prod_{\eta^{\prime} \neq \eta} \hat{R}_{\eta^{\prime}}$ ) and so we can suppose $\alpha: \hat{R} \rightarrow C$. We consider the restriction $\left.\alpha\right|_{R}$. Since $\varphi$ is surjective, there is a $\beta^{\prime}: R \rightarrow F$ with $\varphi \beta^{\prime}=\left.\alpha\right|_{R}$. Since $f \alpha=0$, we see that $\operatorname{Im} \beta^{\prime} \subseteq F^{\prime} \subseteq \operatorname{ker} \phi$. But ker $\phi$ is cotorsion, so there is an $R$-linear $\beta: \hat{R} \rightarrow F$ with $\operatorname{Im} \beta \subseteq$ $\operatorname{ker} \phi$ that agrees with $\beta^{\prime}$ on $R$. Then $\left.(f \varphi \beta)\right|_{R}=\left.(f \alpha)\right|_{R}=0$, and by Proposition 2.1 we get that $f \varphi \beta=0$. This implies $\operatorname{Im} \beta \subseteq F^{\prime}$.

Now that we have that $\varphi: F \rightarrow M_{1}$ is a flat precover, it is easy to argue that it is in fact a flat cover. Since if $d: F \rightarrow F$ is such that $\varphi d=\varphi$, then $f \varphi d=f \varphi$, but $f \varphi=\phi$; so we have $\phi d=\phi$, which implies that $d$ is an automorphism ( $\phi$ is a flat cover). Therefore $\varphi$ is a flat cover and our morphism $f$ is covering.

Acknowledgements. The authors were partly supported by grant CRG 971543 from NATO. J.R.G.R. and L.O. were also supported by grant PB95-1068 from DGES.

\section{References}

1. R. Belshoff, E. Enochs and J. R. García Rozas, Generalized Matlis duality, Proc. Am. Math. Soc. 128 (1999), 1307-1312.

2. L. Bican, R. El Bashir And E. Enochs, All modules have flat covers, Bull. Lond. Math. Soc. (in the press).

3. E. Enochs, Torsion free covering modules, Proc. Am. Math. Soc. 121 (1963), 223-235.

4. E. Enochs, Torsion free covering modules, II, Arch. Math. 22 (1971), 37-52.

5. E. ENOCHS, Injective and flat covers, envelopes and resolvents, Israel J. Math. 39 (1981), 189-209.

6. E. Enochs, Flat covers and flat cotorsion modules, Proc. Am. Math. Soc. 92 (1984), 179-184.

7. E. Enochs, Complete flat modules, Commun. Algebra 23 (1995), 4821-4831.

8. E. Enochs, J. R. García Rozas and L. Oyonarte, Compact coGalois groups, Math. Proc. Camb. Phil. Soc. 128 (2000), 233-244.

9. E. Enochs, J. R. García Rozas and L. Oyonarte, Covering morphisms, Commun. Algebra. 28 (2000), 3823-3835. 
10. E. Enochs, J. R. García Rozas and L. Oyonarte, Are covering (enveloping) morphisms minimal?, Proc. Am. Math. Soc., in press.

11. J. Golan and M. L. Teply, Torsionfree covers, Israel J. Math. 15 (1973), 237-259.

12. J. Gómez AND B. Torrecillas, Torsionfree covers and covers by submodules of flat modules, Commun. Algebra 19 (1991), 803-827.

13. B. Stenström, Rings of quotients (Springer, Berlin, 1975).

14. M. L. Teply, Torsion-free injective modules, Pacific J. Math. 31 (1969), 441-453.

15. M. L. TePly, Torsionfree covers, II, Israel J. Math. 23 (1976), 132-136.

16. M. L. Teply and Seog Hoon Rim, On coverings of modules, Preprint.

17. J. XU, The existence of flat covers over noetherian rings of finite Krull dimension, Proc. Am. Math. Soc. 123 (1995), 27-32.

18. J. Xu, Flat covers of modules, Lecture Notes in Mathematics, vol. 1634 (Springer, Berlin, 1996). 